\title{
Nanostructuring of thin films by ns pulsed laser interference
}

\author{
S. Riedel · M. Schmotz $\cdot$ P. Leiderer $\cdot$ J. Boneberg
}

\begin{abstract}
We show that nanosecond pulsed laser interference can be used to structure surfaces on a nanoscale. With this method, we are able to create hollow structures on various thin films like $\mathrm{Ta}, \mathrm{Ni}, \mathrm{Au}, \mathrm{Cu}, \mathrm{Co}$, and NiTi. We find that the structuring mechanism is related to the mechanical effect of thermal expansion upon melting. To corroborate this model, we study materials with an abnormal behavior at the melting point like $\mathrm{Si}, \mathrm{Ge}$, or $\mathrm{Bi}$, as they contract upon melting.
\end{abstract}

\section{Introduction}

The creation of nanostructures by means of intense laser beams has been studied for several years. Up to now, two types of experiments were performed: experiments with tightly focused beams, and more recently experiments with interference patterns. In the experiments with tightly focused fs pulses, it could be observed that at low intensities hollow bumps were created. At slightly higher energies, nanojets appeared additionally on the bumps [1-5]. Similar results were observed upon fs laser interference experiments with the only difference that a regular pattern of these structures resulted from a single laser shot [6]. Both sets of experiments were reported for thin Au films. While the hollow structure formation in the laser interference experiments was explained by the pressure increase of evaporation at the interface, a further paper discusses another mechanism [7], namely a thermoelastic process which includes thermal expansion and stress formation. Based on this model the authors conclude that this bump formation process is restricted

S. Riedel (§) - M. Schmotz $\cdot$ P. Leiderer - J. Boneberg University of Konstanz, Fach 676, 78457 Konstanz, Germany e-mail: stephen.riedel@uni-konstanz.de to $\mathrm{Cu}, \mathrm{Ag}, \mathrm{Au}$, and very pure metals of the iron family, while other refractory metals should not show such a behavior.

We would like to show here that hollow structures can be generated not only by fs but as well by ns laser pulses. These structures appear on a variety of surfaces, and in particular on metals which have been excluded in [7]. It should be mentioned that such bumps have been observed earlier in focused as well as in interference ns laser experiments on various thin films [8-12], where other mechanisms have been discussed (pressure induced flow, release of compressive stress, Marangoni flow).

\section{Experimental, results and discussion}

We apply ns laser pulses from an injection-seeded Nd:YAG $(\lambda=532 \mathrm{~nm}, \mathrm{FWHM} 10 \mathrm{~ns})$ for the single pulse laser interference lithography (SPLIL). The laser pulse with Gaussian beam profile is split into two or three beams of equal intensity. These beams are then recombined on the substrate under a certain angle of incidence, which defines the period of the interference pattern which shall be produced. Samples used here are thin evaporated metal films of different thicknesses, a $3 \mathrm{~nm} \mathrm{Cr}$ layer served as adhesion layer between the metal film, and the glass substrate. In the case of NiTi, a multilayer consisting of three alternating layers of $50 \mathrm{~nm} \mathrm{Ti}$ and $28 \mathrm{~nm} \mathrm{Ni}$ was prepared. The Ta films were deposited by sputtering without an adhesion layer.

After single pulse illumination with typical laser fluences between $100 \frac{\mathrm{mJ}}{\mathrm{cm}^{2}}$ and $300 \frac{\mathrm{mJ}}{\mathrm{cm}^{2}}$ a periodic pattern appears on the surface. In the experiment, these patterns are observed by the appearance of diffraction from a test laser beam. Then these surfaces are analyzed by optical microscopy, atomic 
force microscopy (AFM), scanning tunneling microscopy (STM), or scanning electron microscopy (SEM). In the latter, an additional focused ion beam (FIB) system allows cutting cross sections through the modulated film at different positions. Due to the Gaussian envelope of the lateral laser beam profile, the intensity dependence of the structuring process can be studied by the examination of the pattern evolution as one moves from the center of the laser spot to its edge.

Above a material-dependent laser threshold, we observe patterns which are shown in Fig. 1 for Ta and NiTi. Similar structures after illumination can be found for the materials $\mathrm{Ni}, \mathrm{Au}, \mathrm{Cu}$, and $\mathrm{Co}$. More examples of other materials will
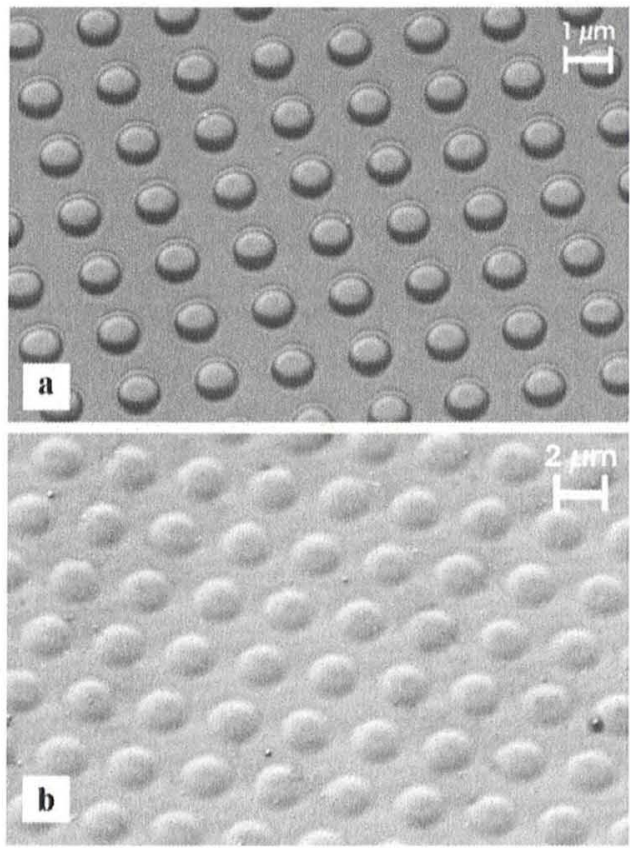

Fig. 1 SEM images of Ta (a) and NiTi (b) surface after three beam interference illumination

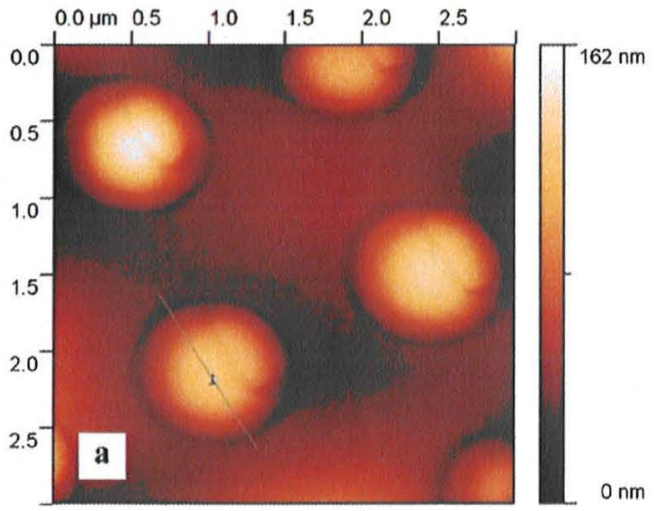

be shown in the next figures. Obviously, the pattern generation is a quite common phenomenon.

As can be seen in the AFM cross section (Fig. 2), the apparent height difference between the tops of the bumps and the surrounding area is about $100 \mathrm{~nm}$ and, therefore, much bigger than the film thickness $(50 \mathrm{~nm})$. Nevertheless, no distinct trench can be observed around the structure. Thus, the suspicion arose that as in the fs experiments hollow microbumps might have emerged. Therefore, the samples were transferred to the electron microscope and analyzed with the focused ion beam.

From the FIB data, we found that indeed the microbump structures are hollow for all the materials mentioned above (Fig. 3). Considering the results described so far the structuring mechanism might be due to the expansion of the film upon melting and the subsequent acceleration off the substrate (in a certain way a similar argument as for the jumping nanodroplets [13]). If this argument is valid, then materials which contract upon melting should show a different behaviour and indeed for thin films of $\mathrm{Si}, \mathrm{Ge}$ and $\mathrm{Bi}$ we observe that no bumps are formed all over the cross section of the laser (Fig. 4). Still ring shaped structures are formed, but these structures appear similar to the illumination of the other materials at high laser intensities, where the structures look like typical dewetting structures [14, 15]. The qualitative appearance of the structures for both groups is independent of the period which we examined $(500 \mathrm{~nm}-$ $3 \mu \mathrm{m})$.

Mechanical stress due to thermal expansion without the necessity of melting was discussed as an alternative structuring mechanism in [7]. We made further experiments to investigate this aspect. For that purpose, we prepared a line pattern by two interfering laser beams. Typically, these lines get hollow at higher intensities. Here, we show the STM pictures at low intensities (Fig. 5). From the cross section, one can see that in this low energy state the bump in the center has a height of $15 \mathrm{~nm}$, while on both sides valleys

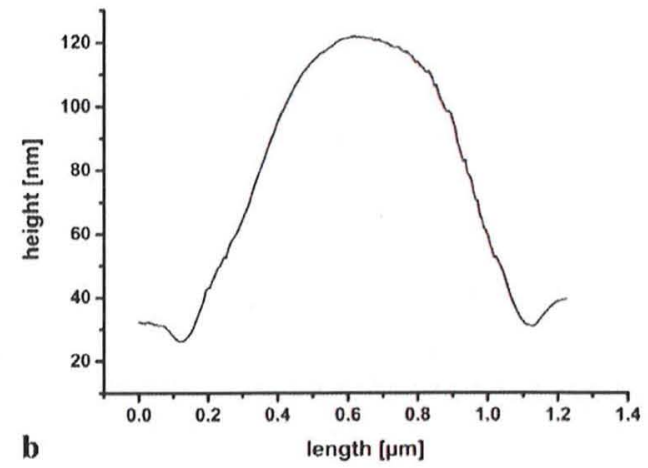

Fig. 2 AFM topography (a) and cross section (b) of microbumps on an illuminated Ta surface. The thickness of the Ta layer was $50 \mathrm{~nm}$. The cross section depicts a height difference of about $100 \mathrm{~nm}$ between the center of the bump and the surrounding area 

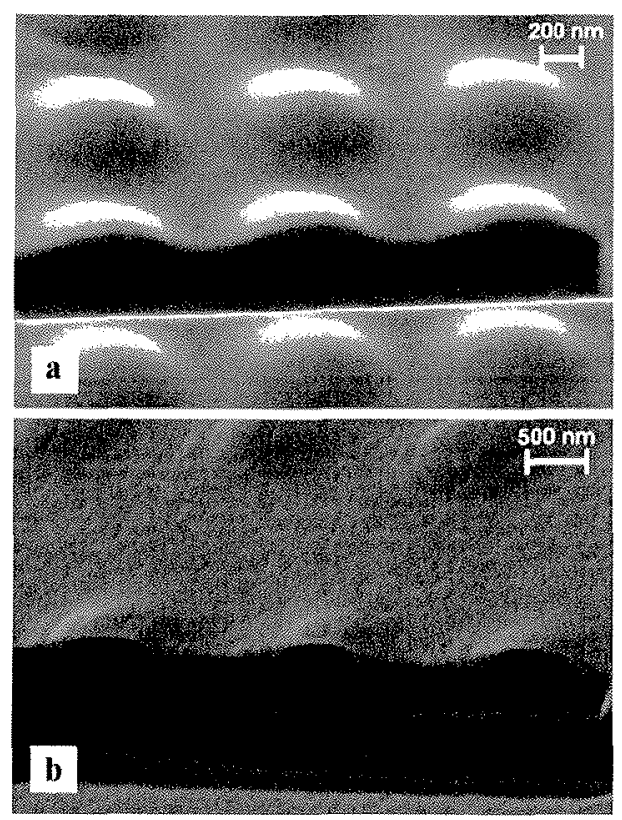

Fig. 3 SEM-images of FIB treated microbumps on Ta (a) and Au (b). The microbumps are hollow
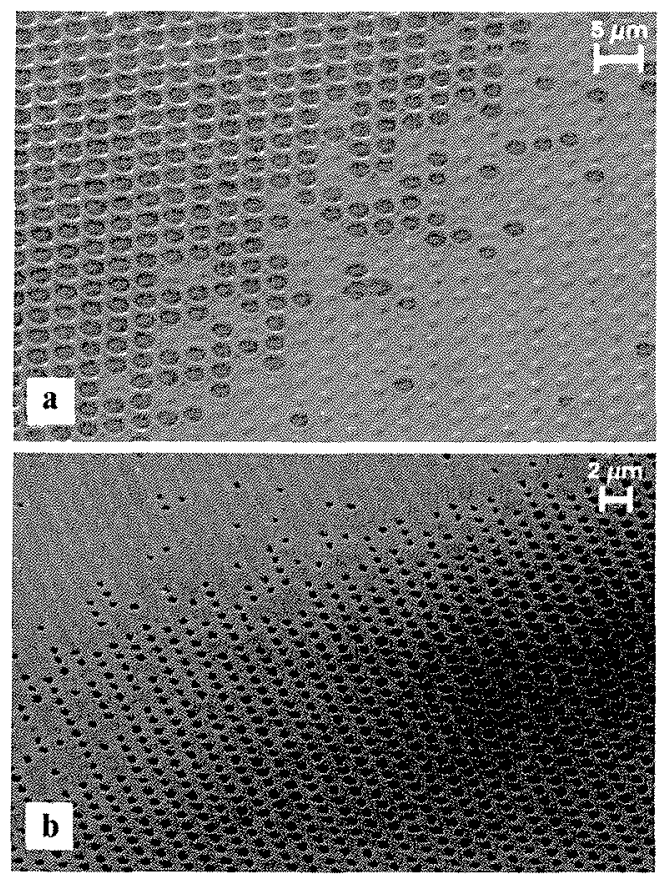

Fig. 4 SEM-images of NiTi (a) and Bi (b) surfaces treated with 3 interfering laser beams. While for the NiTi a transition from bumps to dewetted areas can be observed, on the Bi-surface the microbumps are completely absent

of almost similar depth are formed. One can conclude (and also finds in the FIB-analysis, not shown here) that the structure formed here is not hollow. Nevertheless, one observes that the grain size of the Au across this line is not homogeneous. In order to make this more clear, we made a lineby-line subtraction (Fig. 5, right). Now one can see clearly three different areas in the picture. Outside the line the grain size is small in the order of $50 \mathrm{~nm}$, which is typical for the as grown Au films (A). In the center of the line, the grain size has almost doubled, which can be explained by the melting and subsequent resolidification process $(\mathrm{C})$. At the rims, the grains are not round but elongated towards the center of the rim which shows that lateral growth has taken place (B).

From these observations, we propose the following physical picture for the interaction of nanosecond pulses with thin films: Due to the local temperature increase local stress is building up. Neither this stress nor the upward movement of the center of mass which is induced by the thermal expansion is sufficient to induce a permanent change of topography in our samples. At higher energies, the melting will start in the center of the interference spots, where the intensity is maximal. For most of the metals, this melting process is typically accompanied by a decrease in the density and, therefore, an additional vertical movement of the layer sets in. Due to cohesion, this vertical movement induces also a lateral flow of material. Finally, the liquid layer detaches from the surface. Then the liquid layer might solidify in this delaminated state. At even higher energies, the liquid layer gets unstable. By contrast, the materials which show an increase in density upon melting will experience a flow of material in the direction toward the substrate. Once again, the liquid layer might get unstable and a dewetting process sets in. In comparison with the fs experiments, we could not observe the appearance of jets. This is clear as for fs pulses the melting process is distinctly faster and, therefore, the acceleration of the liquid is higher.

\section{Conclusion}

Our experiments show that the generation of hollow microbumps is directly connected with the change of density upon melting. The liquid gains momentum vertical to the surface, and thus, for metals which show a decrease in density upon melting, the formation of hollow microbumps occurs.

Acknowledgements Financial support by the Center of Applied Photonics (CAP) at the University of Konstanz and the Landesstiftung Baden-Württemberg is gratefully acknowledged. And we thank Matthias Hagner very much for assistance with the focused ion beam system. 
Fig. 5 STM image (left), STM-image with line-by-line subtraction (right) and averaged cross-section (bottom) on a line on $50 \mathrm{~nm} \mathrm{Au}$-film produced by two interfering laser beams
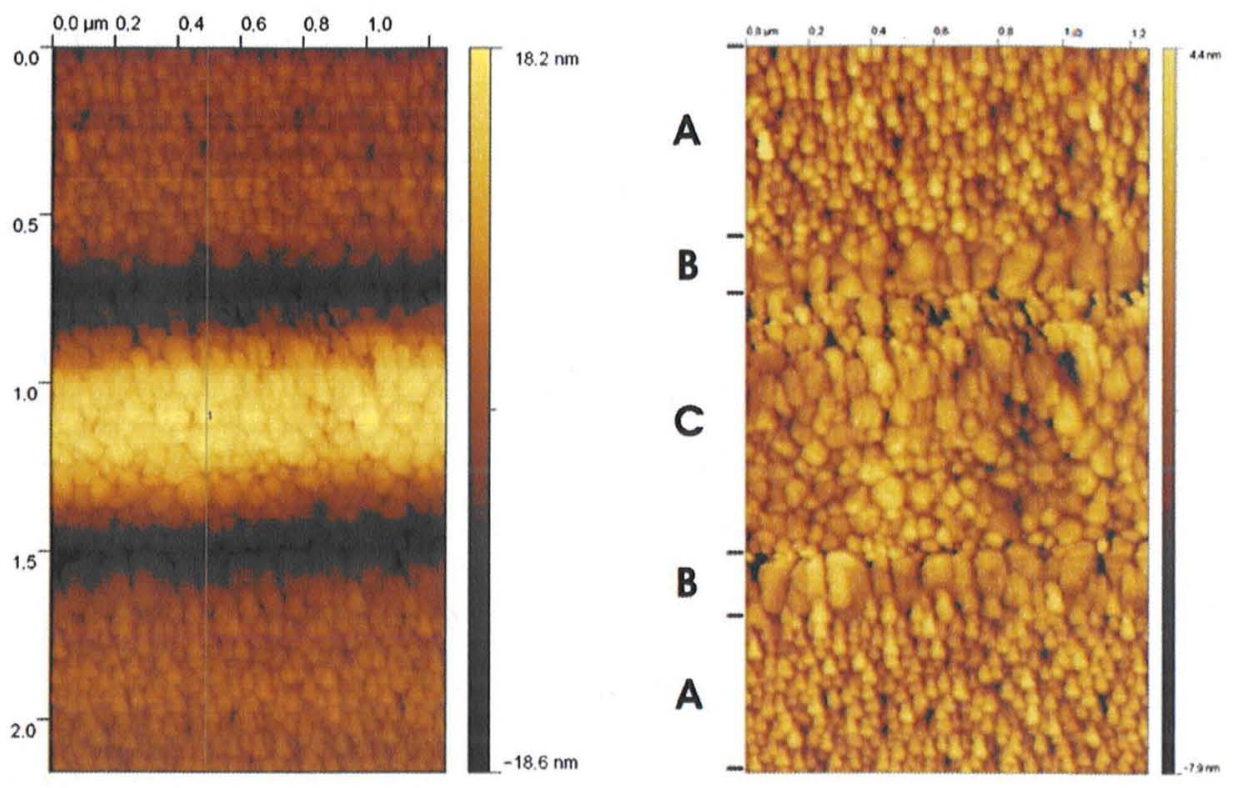

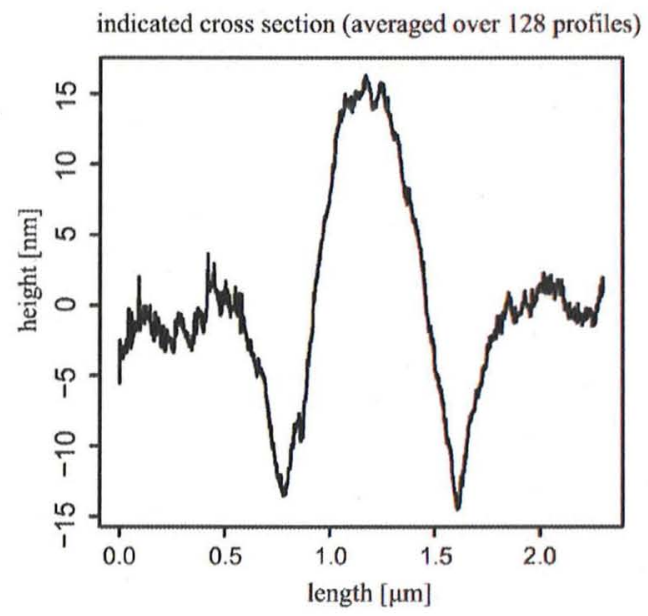

\section{References}

1. A.I. Kuznetsov, J. Koch, B.N. Chichkov, Appl. Phys. A 94, 221 (2009)

2. F. Korte, J. Serbin, J. Koch, A. Egbert, C. Fallnich, A. Ostendorf, B.N. Chivkov, Appl. Phys. A 77, 229 (2003)

3. J. Koch, E. Fadeeva, M. Engelbrecht, C. Ruffert, H.H. Gatzen, A. Ostendorf, B.N. Chivkov, Appl. Phys. A 82, 23 (2006)

4. J. Koch, F. Korte, T. Bauer, C. Fallnich, A. Ostendorf, B.N. Chichkov, Appl. Phys. A 81, 325 (2005)

5. F. Korte, J. Koch, B.N. Chichkov, Appl. Phys. A 79, 879 (2004)

6. Y. Nakata, T. Okada, M. Maeda, Jpn. J. Appl. Phys. 42 (2003)

7. Y.P. Meshcheryakov, N.M. Bulgakova, Appl. Phys. A 82, 363 (2006)
8. K. Xiao, Z.S. Guan, G.J. Wang, L. Jiang, D.B. Zhu, Y.R. Wang, Appl. Phys. Lett. 85, 1934 (2004)

9. F. Mücklich, A. Lasagni, C. Daniel, Intermetallics 13, 437 (2005)

10. A. Lasagni, F. Mücklich, Appl. Surf. Sci. 240, 214 (2005)

11. A. Lasagni, C. Holzapfel, F. Mücklich, Appl. Surf. Sci. 253, 1555 (2006)

12. A. Aktag, S. Michalski, L. Yue, R. Kriby, S. Liou, J. Appl. Phys. 99, 093901 (2006)

13. A. Habenicht, M. Olapinski, F. Burmeister, P. Leiderer, J. Boneberg, Science 309, 2043 (2005)

14. A. Tselev, N. Polushikin, Y. Verevkin, A. Gorbunov, Y. Petryakov, W. Pompe, Appl. Phys. A 69, S819 (1999)

15. Yu. Kaganovskii, H. Vladomirsky, M. Rosenbluh, J. Appl. Phys. 100, 044317 (2006) 